\title{
Withania somnifera shows ability to counter Parkinson's Disease: An Update
}

\author{
Nidhi Singh, Sachchida Nand Rai, Divakar Singh, Surya Pratap Singh* \\ Department of Biochemistry, Institute of Science, Banaras Hindu University, Varanasi - 221005, India
}

Received: June 03, 2015; Accepted: August 07, 2015; Published: October 08, 2015

*Corresponding author: Prof. Surya Pratap Singh, Department of Biochemistry, Institute of Science, Banaras Hindu University, Varanasi- 221005. Email: suryasinghbhu16@gmail.com

\begin{abstract}
Withania somnifera (Ashwagandha) is a very revered herb of the Indian Ayurvedic system of medicine. It is useful for treating various kinds of disease processes and especially used as a nervine tonic. Withania somnifera (Ws) contain a wide array of active components including withaferin A, withanone and other flavonoids exhibiting strong anti-oxidant properties. Many scientific studies on Ws were carried out previously that showed its anti-oxidative effect, synergistic effect with other medicinal herbs and its efficiency to increase catecholamines level and regulation of apoptotic processes. Furthermore, treatment of Parkinsonian mice models with Ws has shown neuroprotection of dopaminergic neurons in substantia nigra pars compacta region of mid-brain. The present review enlightens the crucial role of Indian Ginseng to curb neurodegenerative disorder such as Parkinson's disease. Extensive studies are needed to prove its therapeutic efficacy in neuronal disorders.
\end{abstract}

Keywords: Withania somnifera; Parkinson's disease; neurodegeneration; substantia nigra

\section{Introduction}

Ashwagandha (Withania somnifera, fam. Solanaceae) is popularly known as "Indian Ginseng" or "Indian Winter cherry" $[1,2]$. It is an indigenous medicinal plant exhibiting a vital role in the treatment of various diseases such as stress [3], anxiety [4], arthritis [5] and other disorders related to the central nervous system (CNS) such as Parkinson's [6,7] and Alzheimer's disease [8]. In Ayurveda, the Indian system of medicine, use of herbal plant extracts for treating PD has been well documented by [9] in clinical model. Gupta LG and Rana AC. 2007., [10] stated that whole plant, roots, stem, leaves, seeds and fruits of Ws were used for various experimental studies in order to elucidate their therapeutic applications. Roots of Ws are the main portions of the plant used therapeutically [11]. The dried roots of Ashwagandha are found to be useful in the treatment of nervous and sexual disorders $[11,12]$. The biological activity of Ws extract showed antioxidant and free radical scavenging potential [13]. Additionally, Ws is a potent neuronal tonic and has been expected to be used in the treatment of many neurological deficits including epilepsy [14], poor memory [15], depression [4] and PD [6] in animal model.
Parkinson's disease (PD) is the second most common neurodegenerative disorder after Alzheimer's, affecting 1\% of the population by the age of 65 and $4-5 \%$ of the population by the age of $85[16,17]$. Parkinson's disease is caused by the loss of dopaminergic neurons in the substantia nigra pars compacta region of mid-brain [18] resulting in the reduction of dopamine level [19]. Various factors such as age, genetic and environmental exposure are associated with the onset and progression of PD $[19,20]$.

In this review, we are focussing on the use of therapeutic potential of Withania somnifera (Ashwagandha) in Parkinson's disease. It possesses the power of combating neurodegenerative disorder like PD. Nowadays, more emphasis is laid on the use of medicinal herbs to treat human diseases. Therefore, the cornerstone of this review paper is to focus on how Ashwagandha alleviates Parkinson's disease.

\section{Chemical Constituents}

Rastogi RP and Mehrotra BN 1998.,[21] has revealed over 35 chemical constituents contained in the roots of Ashwagandha through laboratory analysis. The roots of Ws possess withanolides, which are steroidal in nature and bear a resemblance, both in their action and appearance, to the active constituents of the plant Panax ginseng known as ginsenosides [11,12,22]. The biologically active chemical constituents of Ws include alkaloids (isopelletierine, anaferine, cuseohygrine, anahygrine, etc.) and steroidal lactones (withanolides, withaferins) [23]. Ganzera M, Chodhary MI and Khan IA. 2003.,[24] investigated two withanolides of Ws, withaferin A and withanolide D through HPLC analysis. Other constituents of Ws include saponins containing an additional acyl group (sitoindoside VII and VIII), and withanoloides with a glucose at carbon 27 (sitoindoside IX and X) $[24,25]$. Matsuda H, et al.2001, [26] isolated and identified seven new withanolide glycosides of Ws called withanosides I, II, III , IV, V, VI and VII.

\section{Pharmacological effects of Withanolides}

Withania somnifera possesses multiple pharmacological properties which are mainly accredited to the withanolides, its active constituents [7]. Medicinally, Ws root extract is known 
to possess anti-aging [27], anti-oxidant [6] and anti-cancerous properties [28]. Andallu B and Radhika B.2000., [2] demonstrated through clinical observation that roots of Ws are a potential source of hypoglycemic, diuretic and hypocholesterolemic agents. Sitoindosides VII-X and Withaferin-A, the two active principles of Ashwagandha, have been shown to have significant antistress activity when tested in diverse spectrum of stress-induced paradigms [29] and also reported to exhibit strong anti-oxidant activity in rat model [30]. The withanolides serve as hormone precursors that may get converted into human physiologic hormones as needed [22]. According to Ahmed M et al.2005., [31] pretreatment with Ws extract was found to prevent all the changes in antioxidant enzyme activities, catecholamine content, dopaminergic D2 receptor binding and tyrosine hydroxylase expression in PD rat model (Parkinson's induced by 6-hydroxydopamine (6-OHDA)) in a dose-dependent manner. Thus, Ws seems to exert its pharmacological effect by occupying the receptors on the cell membrane and thereby, preventing the binding of actual hormone in a concentration dependent manner. Kobuyama T et al.,2002 [32] illustrated therapeutic potential of withanolide A isolated from root of Ws, that possess ability to regenerate neurites and reconstruct synapses in severly damaged neurons in mice. Amongst withanolides of Ws, withaferin A, a major component of biologically active steroids, showed potent anti-inflammatory [33] and anti- cancerous activity [34]. Thus, the study revealed the pharmacological potential of Ws, which could be harnessed in future, for the therapeutic approaches to PD.

\section{Scientific work done on Withania Somnifera for treating PD}

\section{Effect of Ws on Oxidative stress}

Impaired anti-oxidative defence mechanisms and increased generation of oxidative free radicals, have been implicated in the neurodegenerative conditions like PD. Superoxide dismutase (SOD), catalase (CAT) and glutathione peroxidase (GPX) are the major free radical scavenging enzymes. Defective functioning of these enzymes leads to accumulation of toxic free radicals and consequent degenerative progression of the disease [35]. The active glycowithanolides of Ws were found to elevate the cortical and striatal concentrations of the anti-oxidant enzymes, SOD, CAT and GPX [30]. Manjunath MJ and Murlidhara.2013.,[7] investigated the neuroameliorative effects of Ws in a rotenone (ROT) model of Drosophila melanogaster (Oregon-K). Significant protection was conferred by Ws against ROT-induced lethality, while the survivor flies exhibited improved locomotor phenotype. Furthermore, biochemical investigations revealed that ROTinduced oxidative stress was significantly diminished by Ws. 6-Hydroxydopamine (6-OHDA) is one of the most widely used rat models for Parkinson's disease eliciting its toxic manifestations through oxidative stress. The anti-parkinsonian effect of Ws extract was evaluated and reported to have potent anti-oxidant, anti-peroxidative and free radical quenching properties in various diseased conditions. Ws extract was found to reverse levels of reduced glutathione, GPX, SOD and CAT significantly in a dose-dependent manner as compared to 6-OHDA rat model [31].
Also Prakash J et al.2013., [13] stated through their work about the neuroprotective function of Ws root extract against ManebParaquat (MB-PQ) induced dopaminergic neurodegeneration, in PD mice model. According to their work, Ws extract is capable of inhibiting the oxidative stress occurring in nigrostriatal tissues and simultaneously increasing the count of Tyrosine Hydroxylase positive cells in SN region of the MB-PQ induced PD mice brain. Henceforth, Ws comprehend strong antioxidant potential and its ROS scavenging property plays an important role in the prevention of PD by defying neurodegeneration.

\section{Synergistic Effect of Ws}

Girdhari LG and Avtar CR.2009.,[36] worked on synergistic effect of Ws and L-dopa in the inhibition of haloperidolinduced catalepsy in mice. The anti-cataleptic effect of Ws could be attributed to polyphenols present in it responsible for direct scavenging of free radicals and also by inhibition of lipid peroxidation in the central nervous system. Ws and Mucuna pruriens (Mp) are traditional herbal plants known to have neuroprotective effects due to the presence of L-DOPA in Mp seed powder and withanoloides in Ws root extract [37]. Hence, the synergistic effect of Ws and Mp in Parkinsonian mice induced by chronic exposure to 1-methyl-4-phenyl 1,2,3,6-tetrahydropyridine (MPTP) [38] and Paraquat (PQ), Prakash J et al.2013.,[37] was examined and all the neurochemical variables, oxidative stress and physiological abnormalities were found to be significantly improved compared to untreated PD mice brain. According to Prakash J et al.2013., [37] exposure to $\mathrm{PQ}$ increases nitrite content in the nigrostriatal region. Therefore, they established through their work that Mp + Ws coexposure amends the level of nitrite in PQ treated mice and this decline in nitrite content by $\mathrm{Mp}+$ Ws might be attributed to the antioxidant property of Mp [39] and Ws [1] plant extracts. Also, Malondialdehyde (MDA) a product of lipid peroxidation has been used as a marker of oxidative damage [13]. Prakash J et al.2013., [37] enlightened through their work that after treatment of mice with $\mathrm{PQ}$, the MDA level was highly increased compared to controls. However, MDA levels were significantly ameliorated after the Mp + Ws co-treatment. Thus, the combined treatment of $\mathrm{Mp}+$ Ws showed a significant effect as compared to Mp and Ws treatments alone.

Therefore, the pioneer work done on synergisitic effect of Ws with Mp and Ws with L-Dopa respectively infered about the efficacy of Ws for treating PD.

\section{Effect of Ws on Catecholamines level}

The neurotransmitter, Dopamine (DA) plays a key role in motor control and body movement. Oxidative stress and reduced levels of catecholamines are the contributing factors of neurodegeneration in PD [40] and this leads to the loss of motor function in PD patients [41,42]. RajaSankara S et al.2009., [43] analysed catecholamines such as dopamine (DA), 3,4-dihydroxyphenylacetic acid (DOPAC) and homovanillic acid (HVA) in the Ws treated and untreated PD mice striatum. According to Rajasankara, oral treatment of PD mice with Ws root extract (100 mg/kg body weight) for 7 days or 28 days 
elevated DA, DOPAC and HVA levels in the corpus striatum. Thus, through their work they deduced the medicinal benefit of the Indian traditional herb Ws which enhances catecholamines and antioxidants and prevents lipid peroxidation in the corpus striatum of PD mice. Prakash J et al.2014., [6] studied the effect of Ws on dopamine and its metabolites in the SN region of PD mice. Reduction of dopamine and its metabolites was found in PD mice brain as compared to controls. Further, treatment with Ws for 9 weeks significantly improved dopamine, DOPAC, and HVA levels as compared to untreated PD mice. Hence, it is clear that Ws holds a competency to elevate catecholamines level and fight against PD like disorders.

\section{Effect of Ws on Apoptotic Pathways}

Apoptosis or programmed cell death is a tightly regulated process resulting in the active suicide of cells under a particular set of circumstances. It has been found that one of the main causes of neurodegenerative diseases is the defective regulation of programmed cell death [44]. Bcl-2 is an anti-apoptotic protein that suppresses cell death by inhibiting the action of a proapoptotic protein, Bax. Thus, the Bcl-2 and Bax ratio decides whether a cell will survive or succumb to apoptosis. Interestingly, it has been suggested in a study that over expression of Bcl-2 helps to attenuate MPTP-induced neuronal cell death [45]. Prakash J et al.2014., [6] showed that Bcl-2 expression was significantly down regulated while Bax expression was significantly elevated in a MB-PQ model of PD. Furthermore, it was observed that Ws treatment increased the level of anti-apoptotic (Bcl-2) proteins and decreased the level of the pro-apoptotic (Bax) proteins in the MB-PQ model of PD. Thus, the Indian Ginseng (Ashwagandha) has been emerged with its capability to regulate the level of apoptotic proteins Bcl-2 and Bax respectively. Henceforth, it is clear that Ws owns the ability to overcome neurological disorders like PD.

\section{Discussion and Conclusion}

The present study supports the fact that Ashwagandha is a potent neuroprotective agent and hence, plays a significant role in ameliorating Parkinson's disease, a neurodegenerative disorder. This review paper delineates the potential of Ws to oppose oxidative damage and decline in catecholamines level and also how it exhibits synergistic effect with $\mathrm{Mp}$ and its role in regulation of apoptotic proteins Bcl-2 and Bax. The above discussion clearly outlines the efficiency of Ws to eradicate oxidative stress which is one of the major contributing factor in PD. Thus, the traditional use of Indian Ginseng has a logical and scientific basis which can be exploited in the research area related to PD. Moreover, clinical studies on large scale are needed to prove the efficacy of this herb, especially in PD and other neuronal disorders.

\section{Acknowledgement}

Authors are sincerely thankful to Department of Biochemistry, Institute of Science, Banaras Hindu University, Varanasi for providing us the platform to streamline our basic skill and knowledge in this field and to come up with more innovative ideas in research related to Parkinson's disease in Neurobiology Lab.

\section{References}

1. Singh N, Bhalla M, Jager PD and Gilca M. An Overview on Ashwagandha: A Rasayana (Rejuvenator) of Ayurveda. Afr J Tradit Complement Altern Med.2011; 8(5): 208-213. doi: 10.4314/ajtcam.v8i5S.9.

2. Andallu B and Radhika B. Hypoglycemic diuretic and hypocholesterolemic effect of winter cherry (Withania somnifera Dunal) root. Indian J Exp Biol. 2000; 38(6): 607-609.

3. Bhattacharya A, Ghosal S and Bhattacharya SK. Anti-oxidant effect of Withania somnifera glycowithanolides in chronic footshock stressinduced perturbations of oxidative free radical scavenging enzymes and lipid peroxidation in rat frontal cortex and striatum. Journal of Ethnopharmacology.2001; 74(1):1-6.

4. Bhattacharya SK, Bhattacharya A, Sairam K and Ghosal S. Anxiolyticantidepressant activity of Withania somnifera glycowithanolides: an experimental study. Phytomedicine.2000; 7(6): 463-469.

5. Gupta A ,Singh S. Evaluation of anti-inflammatory effect of Withania somnifera root on collagen-induced arthritis in rats. Pharm Biol.2014; 52(3): 308-320. doi: 10.3109/13880209.2013.835325.

6. Prakash J, Chouhan S, Yadav SK, Westfall S, Rai SN ,Singh SP. Withania somnifera Alleviates Parkinsonian Phenotypes by Inhibiting Apoptotic Pathways in Dopaminergic Neurons. Neurochem Res.2014; 39(12):2527-2536. doi: 10.1007/s11064-014-1443-7.

7. Manjunath MJ, Muralidhara. Standardized extract of Withania somnifera (Ashwagandha) markedly offsets rotenone-induced locomotor deficits, oxidative impairments and neurotoxicity in Drosophila melanogaster. J Food Sci Technol.2013;52(4):1971-81. doi: 10.1007/s13197-013-1219-0.

8. Jayaprakasam B, Padmanabhan K,Nair MG. Withanamides in Withania somnifera Fruit Protect PC-12 Cells from $\beta$-Amyloid Responsible for Alzheimer's Disease. Phytother. Res.2010; 24(6): 859-863. doi: $10.1002 /$ ptr.3033.

9. Nagashayana N, Sankarankutty P, Nampoothiri MR, Mohan PK, Mohanakumar KP. Association of L-DOPA with recovery following ayurveda medication in Parkinson's disease. J Neurol Sci 2000; 176(2):124-127.

10. Gupta LG ,Rana AC. Withania somnifera (Ashwagandha): A Review. Pharmacognosy Reviews.2007; 1(1):129-136.

11. Narinderpal K, Junaid N, Raman B. A Review on Pharmacological Profile of Withania somnifera (Ashwagandha). Research and Reviews: Journal of Botanical Sciences.2013;2:6-14.

12. Singh G, Sharma PK, Dudhe R, Singh S. Biological activities of Withania somnifera. Annals of Biological Research. 2010; 1(3): 56-63.

13. Prakash J, Yadav SK, Chouhan S, Singh SP. Neuroprotective role of Withania somnifera root extract in maneb-paraquat induced mouse model of Parkinsonism. Neurochemical Research.2013;38(5):972-980.

14. Kulkarni SK, Akula KK, Dhir A. Effect of Withania somnifera Dunal root extract against pentylenetetrazol seizure threshold in mice: possible involvement of GABAergic system. Indian J Exp Biol. 2008; 46(6):465469.

15. Baitharu I, Jain V, Deep SN, Hota KB, Hota SK, Prasad D, et al. Withania somnifera root extract ameliorates hypobaric hypoxia induced memory impairment in rats. Journal of Ethnopharmacology.2013; 145(2): 431-441. doi: 10.1016/j.jep.2012.10.063.

16. Eriksen JL, Petrucelli L. Parkinson's disease-molecular mechanisms of disease, Drug Discov. Today: Dis. Mech. 2004; 1(4):399-405. 
17. Singh N, Pillay V,Choonara YE. Advances in the treatment of Parkinson's disease, Prog. Neurobiol.2009; 81(1):29-44.

18. Dauer W, Przedborski S. Parkinson's Disease: Mechanisms and Models. Neuron.2003;39(6):889-909.

19. Singh C, Ahmad I, Kumar A. Pesticides and metals induced Parkinson's disease: involvement of free radicals and oxidative stress. Cell and Molecular Biology.2007;53(5):19-28.

20. Singh MP, Patel S, Dikshit M, Gupta YK. Contribution of genomics and proteomics in understanding the role of modifying factors in Parkinson's disease. Indian J Biochem Biophys.2006; 43(2):69-81.

21. Rastogi RP, Mehrotra BN. Compendium of Indian Medicinal Plants. Central Drug Research Institute. Vol. 6,New Delhi;1998.

22. Verma SK , Kumar A . Therapeutic uses of withania somnifera (ashwagandha) with a note on withanolides and its pharmacological actions. Asian J Pharm Clin Res.2011; 4(1):1-4.

23. Mishra LC, Singh BB , Dagenais S. Scientific basis for the therapeutic use of Withania somnifera (ashwagandha): a review. Alternative Medicine Review.2000; 5(4):334-346.

24. Ganzera M, Choudhary MI, Khan IA. Quantitaive HPLC analysis of withanolides in Withania somnifera. Fitoerapia.2003;74(1-2): 68-76.

25. Elsakka M, Grigoreseu E, Stanescu U, Dorneanu V. New data referring to chemistry of Withania somnifera species. Rev Med Chir Soc Med Nat lasi.1990; 94(2): 358-387.

26. Matsuda H, Murakami T, Kishi A,Yoshikawa M. Structures of withanosides I, II, III, IV, V, VI and VII new withanolide glycosides from the roots of Indian Withania somnifera D and inhibitory activity for tachyphylaxis to clonidine in isolated guineapig ileum. Bioorg Med Chemistry.2001; 9(6):1499- 1507.

27. Babu PVA, Gokulakrishnan A, Dhandayuthabani R, Ameethkhan D, Kumar CVP, Ahamed MI. Protective effect of Withania somnifera (Solanaceae) on collagen glycation and cross-linking. Comparative Biochemistry and Physiology, Part B.2007;147(2):308-313.

28. Khazal KF, Hill DL, Grubbs CJ. Effect of Withania somnifera Root Extract on Spontaneous Estrogen Receptor-Negative Mammary Cancer in MMTV/Neu Mice. Anticancer Res.2014; 34(11): 6327-6332.

29. Bhattacharya SK, Goel RK, Kaur R, Ghosal S. Anti-stress activity of Sitoindosides VII and VIII. New Acylsterylglucosides from Withania somnifera. Phytother Res.1987; 1(1):32-37.

30. Bhattacharya SK, Satyan KS, Ghosal S. Antioxidant activity of glycowithanolides from Withania Somnifera. Indian Journal of Experimental Biology.1997;35(3):236-239.

31. Ahmad M, Saleem S, Ahmad AS, Ansari MA, Yousuf S, Hoda MN, et al. Neuroprotective effects of Withania somnifera on 6-hydroxydopamine induced Parkinsonism in rats. Hum Exp Toxicol .2005; 24(3):137-47.

32. Kuboyama T, Tohda C, Zhao J, Nakamura N, Hattori M, Komatsu K Axon or dendrite predominant outgrowth induced by constituents from Ashwagandha. Neuroreport .2002; 13(14):1715-1720.
33. Sorelle JA, Itoh T, Peng H, Kanak MA, Sugimoto K, Matsumoto S,et al. Withaferin A inhibits pro-inflammatory cytokine-induced damage to islets in culture and following transplantation. Diabetologia.2013; 56(4):814-824. doi: 10.1007/s00125-012-2813-9.

34. Mohan R, Hammers HJ, Bargagna-Mohan P, Zhan XH, Herbstritt CJ, Ruiz A, et al. Withaferin A is a potent inhibitor of angiogenesis. Angiogenesis. 2004; 7(2):115-22.

35. Maxwell SR. Prospects for the use of antioxidants therapies. Drugs.1995; 49(3):345-61.

36. Girdhari LG , Avtar CR. Synergistic effect of Withania somnifera dunal and L-dopa in the inhiition of haloperidol-induced catalepsy in mice. Pharmacognosy Magazine.2009;5(19):46-50.

37. Prakash J, Yadav SK, Chouhan S, Prakash S, Singh SP. Synergistic effect of Mucuna pruriens and Withania somnifera in a paraquat induced Parkinsonian mouse model. Advances in Bioscience and Biotechnology.2013; 4:1-9.

38. Maheswaria T, Vijayrajaa D, Natchiyara RK, Kalaivania K, Rajasankar S, Tamilselvam K, et al. Synergistic Neuropreventive Effect Of Withania Somnifera Root Powder And Mucuna Pruriens Seed Powder In Parkinsonic Mice Model. Journal of Herbal Medicine \& Toxicology.2010; 4(2):63-69.

39. Yadav SK, Prakash J, Chouhan S, Singh SP. Mucuna pruriens seed extract reduces oxidative stress in nigrostriatal tissue and improves neurobehavioral activity in paraquat-induced Parkinsonian mouse model. Neurochemistry International.2013; 62(8):1039-1047. doi: 10.1016/j.neuint.2013.03.015.

40.Surendran S, Rajasankar S. Parkinson's disease: oxidative stress and therapeutic approaches. Neurol Sci. 2010;31(5):531-540. doi: 10.1007/s10072-010-0245-1.

41. Halliwell B. Role of free radicals in neurodegenerative diseases: therapeutic implications for antioxidant treatment. Drugs Aging.2001; 18(9):685-716.

42. Koutsilieri E, Scheller C, Grunblatt E, Nara K, Li J, Riederer P. Free radicals in Parkinson's disease. Journal of Neurology.2002; 249(2):II1-II5.

43. RajaSankar S, Manivasagam T, Sankar V, Prakash S, Muthusamy R, Krishnamurti A,et al. Withania somnifera root extract improves catecholamines and physiological abnormalities seen in a Parkinson's disease model mouse. Journal of Ethnopharmacology.2009; 125(3):369-373. doi: 10.1016/j.jep.2009.08.003.

44. Blum D, Torch S, Lambeng N, Nissou M, Benabid L, Sadoul R, et al. Molecular pathways involved in the neurotoxicity of 6-OHDA, dopamine and MPTP: contribution to the apoptotic theory in Parkinson's disease. Prog Neurobiol.2001; 65(2):135-172.

45. Yang L, Matthews RT, Schulz JB, Klockgether T, Liao AW, Martinou JC, et al. 1-Methyl-4-phenyl-1,2,3,6-tetrahydropyride neurotoxicity is attenuated in mice over expressing Bcl-2. J Neurosci.1998; 18(20):8145-8152. 